\title{
Control System for MR-Guided Cryotherapy - Short-Term Prediction of Therapy Boundary Using Automatic Segmentation and 3D Optical Flow -
}

\author{
Ryoichi Nakamura $^{1,2}$, Kemal Tuncali ${ }^{2}$, Paul R. Morrison ${ }^{2}$, Nobuhiko Hata ${ }^{3}$, \\ Stuart G. Silverman ${ }^{2}$, Ron Kikinis ${ }^{2}$, Ferenc A. Jolesz ${ }^{2}$, and Gary P. Zientara ${ }^{2}$ \\ ${ }^{1}$ Institute of Advanced Biomedical Engineering and Science, Tokyo Women's Medical \\ University, 8-1 Kawada-cho, Shinjuku, Tokyo 162-8666 Tokyo \\ ryoichinaabmes. twmu.ac.jp, \\ ${ }^{2}$ Dept. of Radiology, Brigham \& Women's Hospital and Harvard Medical School, 75 \\ Francis Street, Boston MA 02115 USA \\ \{paul, zentai, worku, kikinis, jolesz, \\ zientara\} @bwh.harvard.edu, \\ ${ }^{3}$ Dept. of Mechano-informatics, Graduate school of Information Science \& Technology, \\ the Univ. of Tokyo, 7-3-1, Hongo, Bunkyo-ku, Tokyo 113-8656 Japan \\ \{ryoichi, noby\}atre.t.u-tokyo.ac.jp
}

\begin{abstract}
During cryotherapy, it is extremely useful for the interventionalists to have available intra-operatively a $3 \mathrm{D}$ iceball visualization, to ensure the effectiveness and safety of the procedure. Additionally, it highly beneficial to provide the interventionalists with a best estimate of how the iceball will grow in the future, and an estimate of the extent to which the target region and the tissues around it will be ablated. In this study, we introduce a newly developed control system for cryotherapy using a novel approach for the real-time/futurepredicted assessments of the treatment. The system has been validated using results from cryotherapy experiments.
\end{abstract}

\section{Introduction}

Cryotherapy (cryocautery, cryosurgery, cryoconization) is the treatment destroying tissue cell by freezing its water component. Cryoprobes containing low temperature circulating liquid are placed in contact with or inside the lesion to be frozen percutaneously. Iceball is created around the tip of the probes and do damage to the tissues. It is percutaneous or interstitials treatment and relatively safe to heat ablation such as laser, RF, so Cryotherapy is very valuable procedure in Minimally Invasive Surgery field. $[1,2]$

But Cryotherapy often suffers from visualization problems in treating internal tumors unless performed under intra-operative image guidance and monitoring due to its less invasiveness [3]. Non-invasive radiological imaging is useful to evaluate soft tissue properties pre-operatively and to monitor changes during surgery. Notably, cryotherapy is interstitial / percutaneous and iceballs are not visible, these imaging technologies are helpful. 
In our institutes, we perform Cryo procedure under interventional MR monitoring system. High quality MR images produce rich information of iceball and its surroundings. But there still be some limitation to use only 2D MR images for monitoring and controlling. At first, it is difficult to detect the accurate iceball shape and visualize in 3D. Secondly, the problem is the inability to estimate the size and geometry of iceball. It is very important to know what will occur for controlling treatments carefully. However it is difficult to predict the future iceballs because radiation absorption and heating is not uniform spatially due to tissue heterogeneity, blood flow, tissue perfusion, etc.

In this paper, we introduce a novel control system for MR-guided cryotherapy. Previously we developed and evaluated a predictive method for estimating the extent and effectiveness of thermal therapies using 2D optical flow [4]. In this study, we expand the method an implement various newly developed modules for 3D visualization, iceball detection and prediction. We also developed several modules for intra-operative assessments for cryotherapy. These modules provide surgeons rich and important information to assess the treatment in real time or predictively. System evaluation and validation was performed using animal cryotherapy experiments data.

\section{Method}

\subsection{Automatic Iceball Segmentation}

The most important module for the control system for cryotherapy is iceball segmentation. It is the base of the software to monitor and validate the thermal effect.

Therapy region on thermal treatment shows tissue intensity changes from light gray (high intensity) to dark gray (low intensity) and finally to black (MR signal void) in MR imaging. Experimental work has shown that within the range of temperatures $37^{\circ} \mathrm{C}$ to $50^{\circ} \mathrm{C} / 37^{\circ} \mathrm{C}$ to $0^{\circ} \mathrm{C} \mathrm{T} 1$ varies approximately linearly with temperature at a rate of $1 \% /{ }^{\circ} \mathrm{C}[5,6]$. I use the percentage of the difference of signal intensities (DiffSI) for the segmentation of thermal changed region. In cryotherapy, Signal intensity increases according to the temperature decreasing in $37 \sim 0^{\circ} \mathrm{C}$. But after starting freezing, intensity changes on another mode.

The DiffSI mapping for thermal region segmentation from T1-w FSE images consist of these steps as described;

1) Image acquisition; access to buffer; data transfer to research workstation

2) FOV definition to enhance the region of interest

3) Image subtraction and division by baseline image

4) Gaussian filtering for noise reduction

5) Adding segmented probe data

6) Segment using region growing method and Image Opening/Closing

The DiffSI-based method is very fast and sensitive, but suffered from SNR. To segment smooth thermal region, we use low-pass Gaussian filtering and image dilation/erosion for noise reduction.

Most of cases in image guided surgery, the lesion is limited and localized region in the whole image field of view (FOV). I use physician-defined FOV for DiffSI mapping to reduce computation load. 
Through those procedure iceball can be segmented, but there are often scattered voxels outside the region due to image noises caused by RF noises, motion of tissues and vessels, and blood flow etc. To cut off the scattered voxels outside of iceball, we use region-growing method for the segmentation. Seed Points for the iceball segmentation must be defined before starting segmentation. But all the therapy regions grow from the tip of the thermal probes (Laser fiber, RF probe, and cryoprobe), hence the
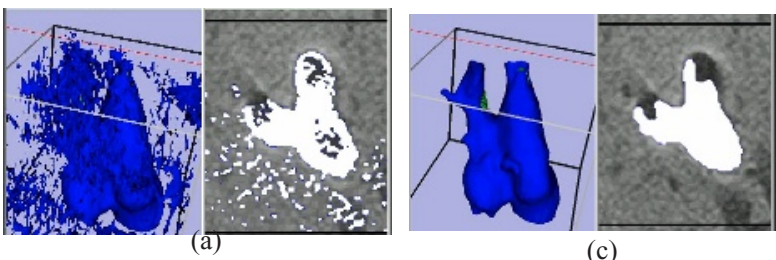

(c)

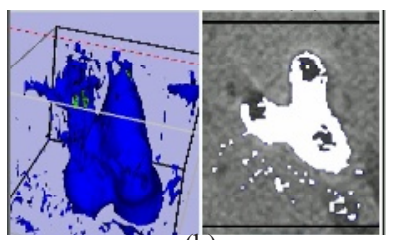

(b)

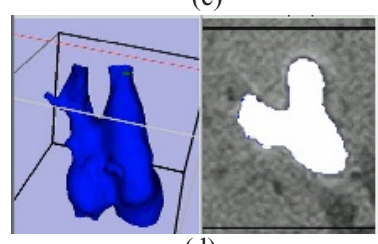

(d)

Fig. 1. Automatic segmentation of iceball. 1): Flowchart of segmentation procedures. r): results of automatic segmentation using Diff-SI image (in-vivo pig experimental result). (a): original DiffSI map. (b): map(a) w/ Gaussian filtering. (c): map(b) + region growing method \& OpenClose Filter. (d): map(c) + segmented probes.

definition of the seed points are not difficult task for the interventionalists.

Finally, adding the segmented crytoprobe volumes, thermal region is created (Fig.1).

\subsection{Predictive Iceball Detection - 3D Optical Flow Estimation -}

Preliminary study, we use 2D optical flow method using second order derivatives [7] and multi-point based solution with least squares (MPLS) [8] for estimating the growing velocities of therapy region [4]. For the fast prediction of therapy effect during treatment, calculation task must be limited enough to update the prediction information near to realtime. Through the advance of computer architecture, we can use powerful CPU and much memory for image processing and visualization.

\section{Optical Flow}

Optical Flow is a mathematical method for estimating the velocity field of voxel motion in the set of images. The gradient-based optical flow constraint equation is described as,

$$
I(x, t)=I(x+v \cdot d t, t+d t)
$$

where $\nabla I(x, t)=\left(I_{s}, I_{y}, I_{z}\right)$ and $I_{t}$ are the $1^{\text {st }}$ order partial derivatives of $\mathrm{I}$.

However, as in two or higher dimensional cases, we cannot solve v. The unknown components of $\mathrm{v}$ are more than two, constrained by only one liner equation. Further constraints or assumptions are necessary to solve for components of v. There are various technique for solve the optical flow constant equation (OFC, $\mathrm{Eq}(1))$. Detailed comparison of those methods appears in [9] .In thermal therapies, the motion of thermal region is diverging (expansion). In the validation on the report [9], Lucas and Kanade's method [10] produced good result. I use this technique for estimating the velocity of thermal region growing, expanding three-dimensional field. 


\section{Prediction Using 3D Optical Flow Information}

When the image dataset at timepoint $\mathrm{n}(\mathrm{t}=\mathrm{t}(\mathrm{n}))$ is taken, 3D optical flow information at timepoint $\mathrm{n}-1$ can be calculated using the image datasets at timepoint n, n-1, n-2. Using the DiffSI image and velocity information (DI(x, $\mathrm{t}(\mathrm{n}-1)), \mathrm{v}(\mathrm{x}, \mathrm{t}(\mathrm{n}-1)))$ at

$$
D I(x+v(x, t(n-1)) \cdot \Delta t, \quad t(n+k))=D I(x, \quad t(n-1))
$$

timepoint $\mathrm{n}-1$, future image at timepoint $\mathrm{n}+\mathrm{k}(\mathrm{t}=\mathrm{t}(\mathrm{n}+\mathrm{k}))$ is estimated using this constant equation;

where $\Delta t=t(n+k)-t(n-1)$.

On cryotherapy, the intra-operative MR images are taken every $2 \sim 3$ minutes but of course the timing of image acquisition is varied. In this study, I use 3 minutes $(180 \mathrm{sec})$ future prediction as the standard length between the timepoint of last image acquisition and the timepoint for prediction. Surgeon can see the iceball on current image dataset and the region on 3 minutes future timepoint on every timepoint.

Using these method and thresholding procedure such as auto-segmentation for actual iceball, future iceball is created.

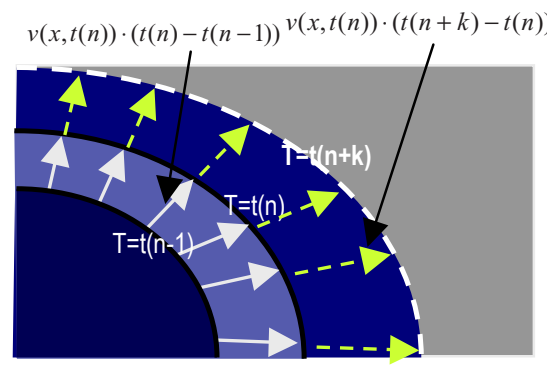

Fig. 2. Prediction method for future iceball. At timepoint $\mathrm{n}$, the velocity of growing of iceball at timepoint $\mathrm{n}-1$ is given by $3 \mathrm{D}$ optical flow computing. On assumption of velocity constancy, the voxel on $x$ at timepoint $\mathrm{n}-1$ will move to $x+v(x, t(n-1)) \cdot \Delta t$.

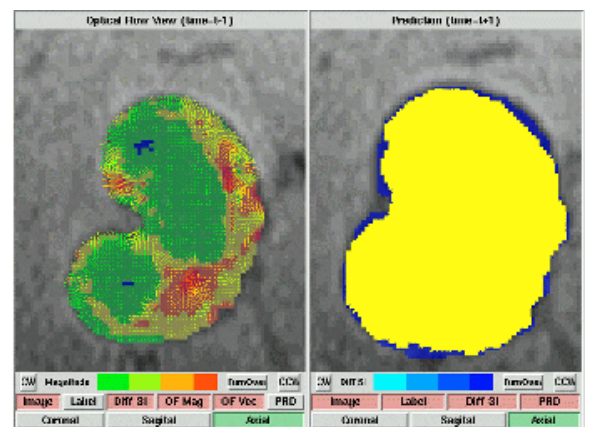

Fig. 3. Optical Flow Estimation and Prediction. Left) Optical Flow vector information is overlaid on the iceball at timepoint t-1. Right) Predicted Iceball Region using the vector information is overlaid the glayscale at $\mathrm{t}+1$. The estimation of iceball region looks pretty good.

\subsection{Module for Assessing Therapy}

By the development of these key features above, we can take accurate and quantitative iceball information. It is very beneficial to assess the treatment in real time. To validate cryotherapy intra-operatively, we first developed and implemented these modules to the software.

\section{- \% Target Coverage (\%TC) and Dice Similarity Coefficient (DSC)}

$\% \mathrm{TC}$ is the most general and simple assessment for measuring the effect of ablation therapies. On this module for thermal therapies, it shows how much target volume is ablated using target volume as volume A, therapy volume as B. In thermal therapies, $\% \mathrm{C}$ must be $100 \%$ at the end of procedure. If not, it leaves residual survival tumor cells. 
DSC shows the similarity between 2 volumes by voxel-by-voxel classification on MRI segmentation dataset. It is generally accepted that a value of DSC $>0.7$ represents excellent agreement [11]

The combination of $\% \mathrm{C}$ DSC shows not only the coverage of target volume by ablation, but also under/over-treatment situation. It is helpful for the treatment validation, including effectiveness and safety [12].

\section{- Alarming module for boundary violation}

Violation of protected region by iceball, like ablating vessels, functional region in brain, means critical damage for patient. To avoid such life-threaten or residual damages, boundary violation must be checked throughout the treatment thoughtfully.

One of the main objectives of development of prediction module for iceball is estimate the situation of boundary violation priory.

\section{- Minimum Ablation Margin}

In ablation therapy, target must be ablated with some marginal zone to avoid residual tumor cells survival. Therefore, it is important message how much the ablation margin is at least. Minimum Ablation Margin module shows to interventionalists where is the minimum marginal area, that is, where is the weakest ablation area.

\subsection{System Overviews}

Fig.4 shows the overviews of the software including the modules described. The viewer window (right on Fig.4) has 4 rendering windows, an alarming window, and a spreadsheet for the assessment of the therapy and 3D optical flow prediction. 4 rendering window show 1) 3D view of therapy area (left-up), 2) 2D slice view of therapy area (right-up), 3) 3D optical flow information on iceball (iceball) (bottom left), and 4) 2D slice view of predicted iceball (bottom right). In 3D window, there are 3 volumes, probes, current iceball, and predicted iceball. Almost of all cases where

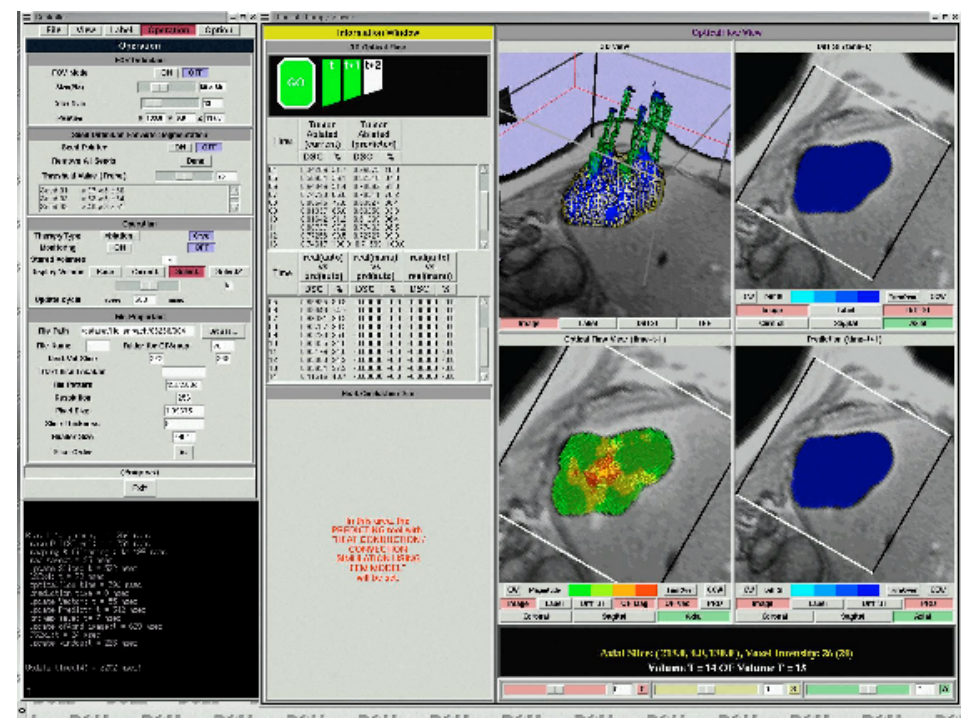

Fig. 4. Overview of the control system for cryotherapy. (Clinical demonstration) 


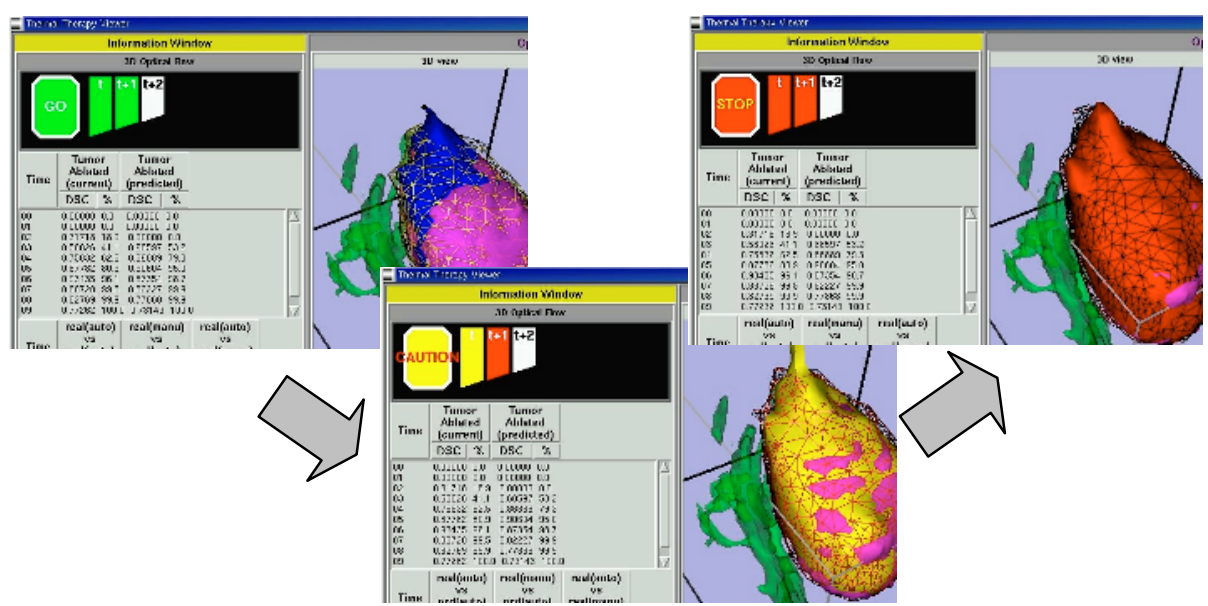

(A)

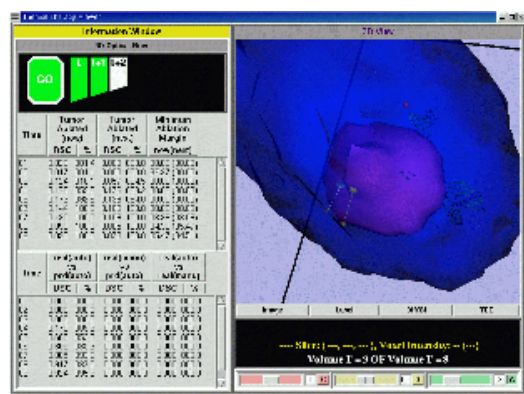

(B)

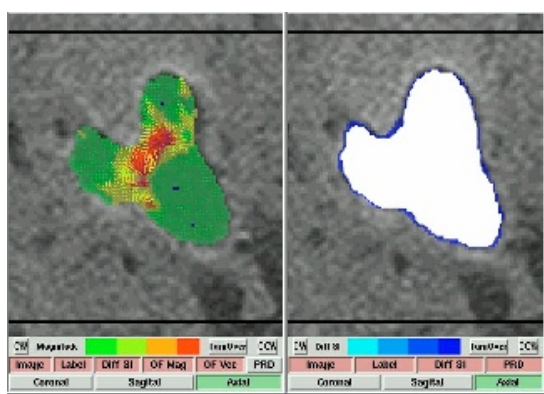

(C)

Fig. 5. Functions of the systems. A) Alarming (Protected boundary violation by auto/predicted), B) Margin (Points \& lines shows the nearest between tumor surface and iceball surface), C) One prediction example in heterogeneous and noisy case.

this software is used, predicted region is larger than current thermal region, hence I use the mesh surface for the predicted region to see the iceball through the mesh.

Figure 5 shows some demonstration of the Functions of this system.

\section{Results}

\subsection{Experimental Design}

The performance of this system mainly depends on the accuracy of auto/predicted iceball detections and the execution speed. We evaluated the accuracy of the segmentation/prediction with the comparison of the generated volumes to manually segmented iceball data which medical experts created. We use 3 in-vivo pig cryo experimental data to validate the accuracy of iceball detection. 
The experimental data are performed under the monitoring using $1.5 \mathrm{~T}$ closed magnet system to have high-resolution datasets for the validation. MR image datasets are taken every 80 second and image acquisition time is 40 second. To evaluate the accuracy of iceball model, we use DSC value with the auto/predicted iceball and manually segmented iceball data by medical experts.

For the assessment of the execution speed, we use several clinical data of cryotherapy and measured the meantime of the execution time.

\subsection{Results}

\section{- Accuracy of auto/predicted iceball detection}

Table 1 shows the results of the comparison between generated auto/predicted iceball and manually segmented iceball data. This evaluation is carried out using all the iceball image data taken during freezing on each experiment. Pig\#1 is the case in which cryoprobes are placed in relatively homogeneous liver tissue and the images have few noises and effects from blood flow. Pig\#2 is the case in which cryoprobes are located to vessel-rich part of the liver and the iceball images are strongly affected vessel movement and blood flow. Pig\#3 is the case at which cryoprobes are located in heterogeneous tissues, such as liver, muscle, and skin. But, these cases have good DSC values of the comparison with manual segmentation data. It shows the high performance of auto/predicted iceball detection module of this control system.

Table 1. DSC comparison results from in-vivo experiments

\begin{tabular}{l|rr|rl} 
& \multicolumn{1}{|c}{ lowest } & highest & average & \multicolumn{1}{c}{ sd } \\
\hline \hline pig1:auto segmente & 0.758 & 0.957 & $\mathbf{0 . 9 3 7}$ & $\mathbf{0 . 0 5 1}$ \\
pig2:auto segmente & 0.748 & 0.994 & $\mathbf{0 . 9 3 0}$ & $\mathbf{0 . 0 6 3}$ \\
pig3:auto segmented & 0.850 & 0.996 & $\mathbf{0 . 9 0 7}$ & $\mathbf{0 . 0 3 9}$ \\
pig1:predicted & 0.736 & 0.954 & $\mathbf{0 . 9 0 7}$ & $\mathbf{0 . 0 7 2}$ \\
pig2:predicted & 0.661 & 0.938 & $\mathbf{0 . 8 7 0}$ & $\mathbf{0 . 1 0 6}$ \\
pig3:predicted & 0.735 & 0.930 & $\mathbf{0 . 8 7 1}$ & $\mathbf{0 . 0 6 2}$
\end{tabular}

We also checked if the performance of iceball prediction is grater than other methods including 2D optical flow, Uras' method. The system we newly developed has significant improvements in accuracy of the prediction.

\section{- Execution time in clinical environment}

We evaluate the performance of the guidance information updating.

This evaluation is done using some of the actual therapy data because the pig data used in accuracy evaluation have higher resolution than ordinal clinical cases. Using 256x256x8 20 voxel slab (256x256resolution, 8 20slices for each timepoint) and $64 \times 64 \times 20 F O V$ for computation region around cryoprobes, all the update is carried out in approx $2.5 \mathrm{sec}$. Most of clinical cases, it takes about 1 minute for this kind of images on T1-w FSE protocol using 0.5T Interventional MRI. Therefore, this result shows the novel performance of this control system for cryotherapy. 


\section{Discussion and Conclusions}

We developed a novel MRI-based monitoring and control system for cryotherapy. The system shows the interventionalists rich visual and quantitative information of the treatment in real-time and provides highly beneficial predicted information regarding the course of the treatment. Two key components of the system provide accurate quantitative iceball information and the 3D visible model.

Results of animal experiments were used to demonstrate the good accuracy of the system. Noisy, or heterogeneous cases are The results from using our system during animal cryotherapy experiments indicate the system's value in increasing the therapeutic effectiveness of the ablation and the safety of the procedure. We now improve and evaluate the system performance using 1) several different methods for iceball segmentation, and 2) estimation of more accurate "true" iceball shape from the sets of hand segmentation results for more reliable assessment. We are also attempting to assess its clinical ability in real operation field in direct system connection to MR system.

Acknowledgements. This research is supported by NIH R01-CA86879, Harvard University Milton Fund, and Grant-Aid for Research Fellow of the Japanese Society for the Promotion of Science.

\section{References}

1. Balmer A, Gaillound C, De Potter P, Munier F, Chamero J, Treatment of retinoblastoma and results, Lausanne1963-1989 Klin Monatsbl Augenheilkd 1990 May, 196(5), pp.374376,1990

2. Tsuji T, Otake N, Nishimura M, Cryosurgery and topical fluorouracil: a treatment method for widespread basal cell epithelioma in basal cell nevus syndrome, Journal of Dermatology, 20(8), pp507-513, 1993

3. Muller Lisse UG, Heuck AF, Control and monitoring of focal thermotherapy with magnetic resonance tomography. An overview, Radiology, 38(3), pp.200-209, 1998

4. Zientara GP, Saiviroonporn P, Morrison PR, Fried MP, Hushek SG, Kikinis R, Jolesz FA. MRI Monitoring of Laser Ablation Using Optical Flow, Journal of Magnetic Resonance Imaging, No.8, pp1306-1318, 1998

5. Matsumoto R, Oshio K, Jolesz FA. T1-weighted MR monitoring for interstitial laser- and freezing-induced ablation in the liver, Journal of Magnetic Resonance Imaging, No2, pp555-562, 1992

6. Kuroda K, Chung AH, Hynynen K, Jolesz FA. Calibration of water proton chemical shift with temperature for non-invasive temperature imaging during focused ultrasound surgery, Journal of Magnetic Resonance, No.8, pp175-181, 1998

7. Uras S, Girosi F, Torre V, A Computational Approach to Motion Perception, Biological Cybernetics, vol. 60, pp. 79-87, 1988.

8. Nesi P, BimboD, Ben-Tzvi D. Algorithms for Optical Flow Estimation in Real-time on Connection Machine-2, Department of Systems and Informatics Technical Report, Faculty of Engineering University of Florence Italy, DSI-RT 24/92, 1992

9. Barron JL, Fleet DJ, Beauchemin SS. Performance of Optical Flow Techniques, International Journal of Computer Vision, No.12(1), pp43-77, 1994 
10. Lucas B, Kanade T. An Iterative Image Registration Technique with an Application to Stereo Vision, Proc of DARPA Image Understanding Workshop, pp121-130, 1981

11. Bharatha A, Hirose M, Hata N, Warfield SK, Ferrant M, Zou KH, Suarez-Santana E, Ruiz-Alzola J, D'Amico A, Cormack RA, Kikinis R, Jolesz FA, Tempany CMC. Evaluation of three-dimensional finite element-based deformable registration of pre- and intra-operative prostate imaging., Medical Physics, No28, pp2551-2560, 2001.

12. Tuncali K, Morris M, Warfield SK, Morrison PR, Dahm F, Shankar S, Sonnenberg E, Silverman SG. 3D Assessment of Percutaneous MRI-guided Cryotherapy of Liver Tumors, IVth Interventional MRI Symposium of ISMRM, Leipzig Germany, September 2002 